\title{
Adsorption and Wetting Mechanisms at the Surface of Aqueous Hydrocarbon Solutions as a Possible Source of Atmospheric Pollution
}

\author{
M. Sadiki ${ }^{1}$, F. Quentel ${ }^{1}$, C. Elléouet ${ }^{1}$, R. Olier ${ }^{1}$ and M. Privat ${ }^{1 *}$ \\ 1 UMR CNRS 6521, Département de chimie, Université de Bretagne Occidentale, \\ 6, avenue Le Gorgeu, C.S. 93837, 29238 Brest Cedex 3 - France \\ e-mail: mustapha.sadiki@univ-brest.fr - francois.quentel@univ-brest.fr - catherine.elleouet@univ-brest.fr \\ rene.olier@univ-brest.fr-mireille.priva@@univ-brest.fr \\ *Corresponding author
}

\begin{abstract}
Résumé - Mécanismes d'adsorption et de mouillage à la surface de solutions aqueuses d'hydrocarbures comme source possible de pollution atmosphérique - Les solutions d'hydrocarbures ne sont pas très souvent étudiées. Cependant, leur comportement est impliqué dans de nombreux mécanismes, en particulier les mécanismes environnementaux. Dans le présent article, nous présentons la synthèse de plusieurs travaux ayant trait au comportement superficiel, en particulier d'adsorption, de solutions non saturées de benzène et de cyclohexane. Il y est mis en évidence la coadsorption de nitrate de plomb, bien que celui-ci, en l'absence de molécules organiques dans la surface, ne s'adsorbe pas du tout. Ces données ont été obtenues essentiellement au moyen d'une technique rarement utilisée, la colonne à bulles, brièvement décrite ci-dessous. Celle-ci, en dépit des contraintes de sa mise en œuvre, s'est révélée très utile dans l'étude de ces composés faiblement adsorbables, et peu actifs sur la tension de surface. L'étude des mélanges se fait directement, sans avoir à utiliser de modèle, ce qui est précieux pour des études environnementales. Les résultats obtenus établissent de façon convaincante que des mécanismes, tels que le pétillement, joints à la coadsorption, peuvent entrainer le passage de composés nocifs pour l'environnement, peu solubles, peu actifs sur la surface quand ils sont isolés, depuis l'eau jusque dans l'atmosphère.
\end{abstract}

\footnotetext{
Abstract - Adsorption and Wetting Mechanisms at the Surface of Aqueous Hydrocarbon Solutions as a Possible Source of Atmospheric Pollution - Hydrocarbons in solutions have been the subject of very few investigations despite their relevance, in particular, in situations where environmental mechanisms are involved.

We present, here, a synthesis of several studies conducted within our laboratory about the adsorption, at the water surface, of benzene and cyclohexane from under-saturated solutions. The coadsorption of lead nitrate is also evidenced, though it does not adsorb in absence of organic molecules in the surface. Most of the data reported here were collected from series of measurements made with a very uncommon method: the bubble column; this technique, though being very time-demanding and lacking of userfriendliness, proved its usefulness and relevance in the study of such weakly adsorbable, surface-tension inactive compounds. The study of mixtures is simple and requires no model, which is precious in environmental research. The gathered data demonstrate that, through mechanical mechanisms such as bubbling, coadsorption can lead to the passing from water to the atmosphere of harmful non-soluble, poorly surface-active, components.
} 


\section{LIST OF MAIN SYMBOLS}

$\alpha$ vapour phase in equilibrium with the liquid phase(s).

$\beta \quad$ water-rich liquid phase.

$\gamma$ hydrocarbon-rich liquid phase.

$\hat{\gamma}$ contact angle in a drop of $\gamma$ lying on $\beta$ phase in contact with $\alpha$ phase.

J solute concentration profile in the bubble column.

$J_{i}$ solute concentration profile of $i$ component, in the bubble column.

$\mathbf{K}$ proportionality constant in an adsorption isotherm of Freundlich type.

$K_{i}$ proportionality constant in adsorption isotherm of $i$ component (Freundlich type).

\section{INTRODUCTION}

It is commonly admitted that the light hydrocarbons present in the atmosphere [1] come from industrial activities, road traffic, or evaporation from accidentally-spread layers. But, the contribution of the atmospheric dissolution of such species through vapour pressure equilibrium or surface adsorption and bubbling has been rarely considered in atmospheric pollution mechanisms [2-4]. However, in the vicinity of wrecked tankers with huge amounts of oil progressively leaking from their tanks and dissolving in the seawater, the passing of hydrocarbons from water to the atmosphere through surface mechanisms is worth being taken into account. This is also true near industrial wastelands where interstitial waters charged with dissolved hydrocarbons may feed with them ground waters, springs and surface waters. Moreover, adsorption is of a much greater concern whenever the presence of hydrocarbons induces the coadsorption of other harmful species unable to adsorb and be transferred otherwise.

In previous studies [5-6], we showed that, once adsorbed at the air/water interface, benzene and cyclohexane can induce the coadsorption of a heavy metal salt, namely lead nitrate. We selected this salt because of its solubility properties; in addition, it constitutes an excellent model for ions of concern in environmental problems [7]. The present report focuses on the specific role played by hydrocarbons in the experimental data collected over our experiments; it also gives more insight into the specific method imposed by the nature of our investigations.

Indeed, the study of hydrocarbon adsorption is uneasy because the method classically used to get air/water adsorption relies on the treatment of surface tension data by applying the Gibbs isotherm formula. Unfortunately, despite the well-established adsorption of hydrocarbons in noticeable amounts [9], the very low activity demonstrated by most of them on water surface tension imposes the use of different methods for studying surface properties. For instance, the wetting of hydrocarbons has been investigated by ellipsometry [10-12].

However, in the study of benzene in solutions reported here the first parameter to be measured was surface tension because it can, conversely to other hydrocarbons, be determined close to the solubility limits. On the other hand, for very dilute solutions and hexane we applied the bubble column method [13], which is in fact a variant of the depletion method used to study adsorption on solid suspensions; in the case of a three-component mixture, this method allows one to determine the adsorption of each solute. But, since its implementation is quite difficult, it is worth being employed only in very specific cases where no other method is relevant. Whenever surface tensions are employed for three-component mixtures, one must be aware that their use requires that Gibb's formula be written in a convenient form. The study of coadsorption is then a rather difficult task, however simplified in dilute solutions, which is the case here. As the mutual wetting properties of water-rich and hydrocarbon-rich phases in equilibrium at the solubility limit are now known to significantly affect adsorption process [14-15], the first part of this study will introduce such data.

\section{THEORETICAL ASPECTS}

\subsection{Adsorption and Wetting}

Cahn's theory [14-16] shows that adsorption layers and complete wetting can be described in terms of phase nucleation. This is particularly valuable near a critical point where one of the coexisting phases completely wets the other one at the contact with vapour.

The $B$ and $C$ phase diagrams presented on Figure 1 are characteristic of binary systems each of them composed of two partly miscible liquids (e.g. water and hydrocarbon); these systems exhibit a wetting transition at a temperature $T_{W}$, and $x$ is the molar fraction of the hydrocarbon. Waterrich and hydrocarbon-rich solutions are respectively on the left and right sides of the diagram; solutions of both types coexist within the miscibility gap. Conversely to $A$ diagram (which could be, for instance, nicotine/water or dimethylpyridine/water diagram) lower critical points are obviously missing on $B$ and $C$ diagrams; however, the shapes of both diagrams suggest that their occurrence has been prevented by crystallisation.

On this figure, it is worth focusing on the physical processes occurring along a horizontal path (at $T<T_{W}$ ) starting from the water-rich phase $(\beta)$ below the wetting transition temperature: indeed, while moving closer to the coexistence line, the surface layer is progressively enriched in hydrocarbon until its composition and that of the $\gamma$ phase become alike. The $\beta$ phase is then totally wetted by the forming hydrocarbon-rich phase $(\gamma)$. Because of similarities 
between the nearly alike compositions of $\beta$ and $\gamma$ phases, most of the time this complete wetting is observed near the critical point (whenever it exists); let us denote $\alpha$ the vapour, then the relation between the different surface tensions can be expressed as follows [16]:

$$
\sigma_{\beta \alpha}=\sigma_{\beta \gamma}+\sigma_{\gamma \alpha}
$$

This situation corresponds to a null spreading coefficient, $S=\sigma_{\beta \alpha}-\sigma_{\gamma \alpha}-\sigma_{\beta \gamma}=0$.

Far from the critical point, relation (1) becomes an inequality:

$$
\sigma_{\beta \alpha}>\sigma_{\beta \gamma}+\sigma_{\gamma \alpha}
$$

This inequality always corresponds to the complete wetting of $\beta$ by $\gamma$ but, here, it corresponds to a positive spreading coefficient, $S=\sigma_{\beta \alpha}-\sigma_{\gamma \alpha}-\sigma_{\beta \gamma}>0$. It is worth recalling that spreading coefficients are positive, negative or null depending on their definitions and on the wetting properties of the system under study [17-19].

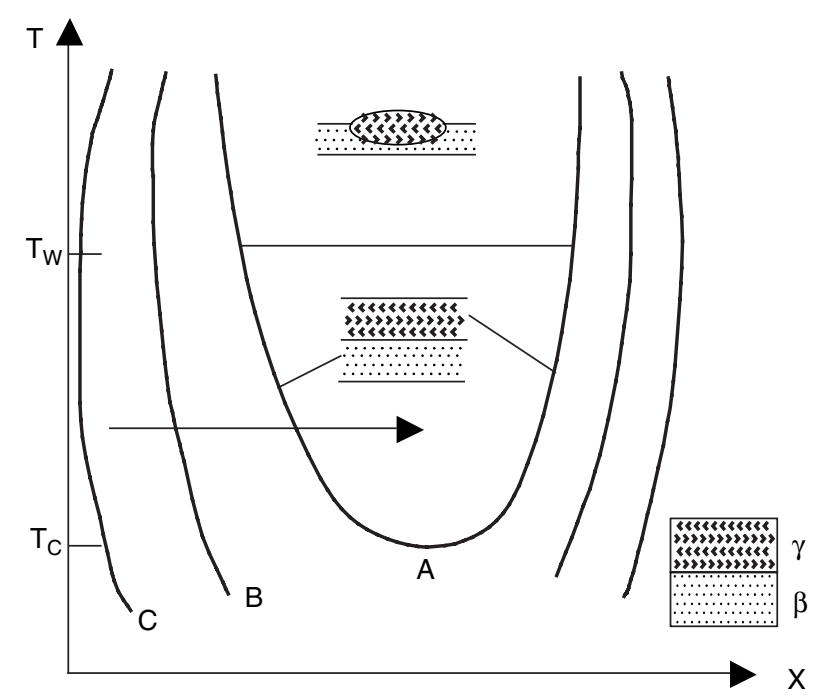

Figure 1

Phase behaviour of hydrocarbon-water mixtures. Parts $C$ and $B$ are schematic, but realistic representations of cyclohexane and benzene diagrams respectively, in the range $0-80^{\circ} \mathrm{C}$. Part $A$ is a complete phase diagram where the lower critical point can be observed. Along the coexistence curve, the water-rich $\beta$ phase and hydrocarbon-rich $\gamma$ phase in equilibrium exhibit a wetting behaviour against the $\alpha$ vapour phase. Wetting depends on temperature and is shown at the centre of the figure. Close to $T_{C}$, wetting of $\beta$ by $\gamma$ is complete. $T_{W}$ is the wetting transition temperature, above which wetting is partial. Neither B diagram nor $\mathrm{C}$ one exhibit a lower critical point, but their shapes suggest the existence of a lower consolute point, which would have been visible if crystallisation had not interrupted them.
But, one should note that the above reasoning about adsorption (see Fig. 1) is always valid under complete wetting condition and whatever the relation (1) or (2) between the surface tensions.

The reverse inequality:

$$
\sigma_{\beta \alpha}<\sigma_{\beta \gamma}+\sigma_{\gamma \alpha}
$$

corresponds to a partial wetting of $\beta$ by $\gamma$. Let us consider a drop from $\gamma$-phase and denote $\hat{\gamma}$ the angle between the tangents to the three interfaces at the triple contact line; this angle is related to interfacial tensions by [16]:

$$
M=\frac{\left[\left(\sigma_{\beta \alpha}\right)^{2}-\left(\sigma_{\gamma \alpha}\right)^{2}-\left(\sigma_{\beta \gamma}\right)^{2}\right]}{\left[2 \sigma_{\gamma \alpha} \sigma_{\beta \gamma}\right]}(=\cos \hat{\gamma})
$$

where $M$ is the wetting coefficient of $\beta$ phase by $\gamma$ phase in contact with $\alpha$. M equals cos $\hat{\gamma}$ only when Equation (3) is proved. Relation 4 is the equivalent of the Young law for the wetting of solids. Equation (2) corresponds to a wetting coefficient $M>1$. Passing from complete to partial wetting constitutes a wetting transition, which affects adsorption behaviour.

\subsection{Bubble Column Theory}

In the bubble column filled with the solution under study, numerous rising bubbles of small size carry upwards the species adsorbed at their surface, and then burst at the top (Fig. 2). The resulting concentration gradient is analysed at the time when the steady state is established.

According to the theory developed by Lemlich [20] and applied by Wan and Tokunaga [13], the fundamental hypothesis is Equation (5) where $\mathbf{K}$ is the proportionality constant; this relation assumes a linear variation of adsorption, $\Gamma$, versus the bulk concentration, $C$. In environmental studies [21], this isotherm equation is often termed the Freundlich isotherm (with exponent parameter equal to 1); but, in fact it is rather the limit form of the Langmuir isotherm at very low concentrations [8]. This approximate form can be used for almost all of the systems with very small adsorption values:

$$
\Gamma=\mathbf{K} C
$$

In this report, for better readability, the factor $\mathbf{K}$ will be named later "adsorption coefficient".

At steady state and with respect to the species concentration at the column bottom, $C_{b}$, the concentration profile $C(z)$ along the column is:

$$
\frac{C(z)}{C_{b}}=\exp (\mathbf{J} z)
$$

with

$$
\mathbf{J}=\frac{a f \mathbf{K}}{A D}
$$




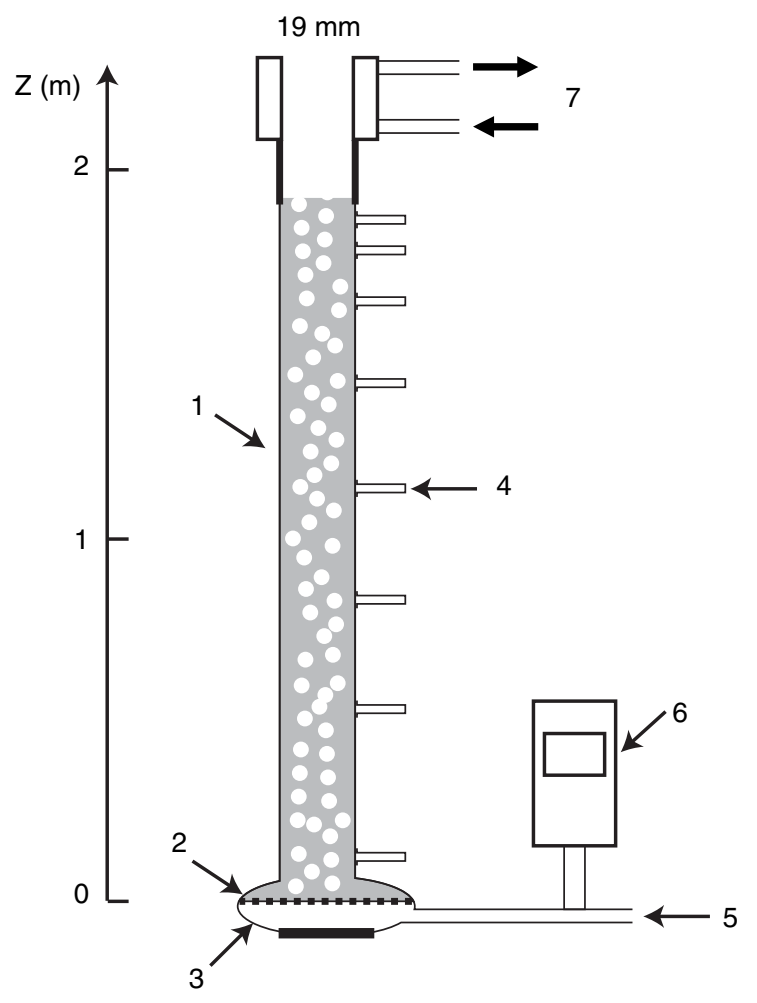

Figure 2

Bubble column device.

1. Bubble column; 2. Fritted glass plate; 3. Air chamber;

4. Sampling ports; 5 . Air inlet; 6. Mass flow controller;

7. Cooler used to trap vapour.

where $A$ is the column cross-section, a is the surface area per bubble, $f$ is the bubble generation rate (calculated from the measured gas flow and the bubble volume mean value), and $D$ is the eddy dispersion coefficient; moreover, the gas within each bubble is supposed to be at atmospheric pressure. The parameter $\mathbf{J}$ characterises the separation of the solute from the solvent induced by the adsorption upon the ascending bubbles.

Moreover, integrating Equation (6) along the column height and expressing the mass conservation during the building of concentration gradient lead finally to:

$$
\frac{C(z)}{C_{0}}=\left[\frac{H \mathbf{J}}{\exp (H \mathbf{J})-1}\right] \exp (\mathbf{J} z)
$$

where $C_{0}$ is the species homogeneous initial concentration, and $H$ is the height of the liquid column [13]. For each species present in the bubble column, the measurement of $C(z)$ at steady state along the column versus the altitude $z$ gives $\mathbf{J}$ from Equation (8). With this value of $\mathbf{J}$, additional measurements of $A, a, f$ and $D$ lead to $\mathbf{K}$ by solving equation (7), and finally to $\Gamma$ by using Equation (5). Usually,
$D$ can be determined from the expression of the timedependent concentration profile and measurement of such profiles by different methods $[13,22]$. Actually, we followed Wan and Tokunaga and took always the same value of $D$ $\left(6.1 \times 10^{-4} \mathrm{~m} \mathrm{~s}^{-1}\right)$ and the same relative uncertainty, 0.1 , because the characteristics of our column and system were alike theirs.

\subsection{Adsorption from Surface Tension in the Case of Two Solutes}

\subsubsection{Lead Nitrate Surface Content in Presence of Hydrocarbon (Benzene)}

Let us consider a system of surface tension $\sigma$ containing a surface layer; it consists of a salt (denoted s), composed of cations (+) and anions (-), benzene (B) and water (W). Application of Gibbs Duhem's relation gives:

$$
-d \sigma=\Gamma_{+} d \mu_{+}+\Gamma_{-} d \mu_{-}+\Gamma_{B} d \mu_{B}+\Gamma_{W} d \mu_{W}
$$

where $\Gamma_{i}$ is the adsorption of $i$ species.

Gibbs Duhem's equation for the bulk writes:

$$
x_{+} d \mu_{+}+x_{-} d \mu_{-}+x_{B} d \mu_{B}+x_{W} d \mu_{W}=0
$$

where $x_{i}$ is i mole fraction.

Eliminating $d \mu_{W}$ between (9) and (10) leads to Gibbs' equation:

$$
-d \sigma=\Gamma_{+, W} d \mu_{+}+\Gamma_{-, W} d \mu_{-}+\Gamma_{B, W} d \mu_{B}
$$

where $\Gamma_{i, w}$ is the relative adsorption of $i$ component with respect to water,

$$
\Gamma_{i, W}=\Gamma_{i}-\left(\frac{x_{i}}{x_{W}}\right) \Gamma_{W}
$$

Let us write $\Gamma_{+, W}=v_{+} \Gamma_{S, W}$ and $\Gamma_{-, W}=v_{-} \Gamma_{S, W}$ where $v_{+}$and $v_{-}$are the number of cations and anions in one molecule of salt, and then:

$$
d \mu_{S}=v_{+} d \mu_{+}+v_{-} d \mu_{-}
$$

Gibbs' equation becomes:

$$
-d \sigma=\Gamma_{S, W} d \mu_{S}+\Gamma_{B, W} d \mu_{B}
$$

With lead nitrate, $v_{+}=1, v_{-}=2$, and the corresponding term $v=v_{+}+v_{-}=3$.

The next step is the evaluation of $d \mu_{B}$ as it may manifest in an experiment where the molarity $c_{B}$ has been kept constant contrarily to the formality $c_{S}$. All chemical potentials in the solution are related through the bulk Gibbs Duhem equation (10), which writes:

$$
x_{S} d \mu_{S}+x_{B} d \mu_{B}+x_{W} d \mu_{W}=0
$$


where:

$$
\mu_{S}=g_{S}^{0}+R T \ln \left[\gamma_{+}{ }^{v_{+}} \gamma_{-}{ }^{v_{-}}{v_{+}}^{v_{+}} v_{-}{ }^{{ }}{ }_{-} c_{S}{ }^{\left(v_{+}+v_{-}\right)}\right]
$$

or:

$$
\mu_{S}=g_{S}^{0}+R T \ln \left[\gamma_{ \pm}{ }^{v} v_{+} v_{+} v_{-}{ }^{v_{-}} c_{S}{ }^{v}\right]
$$

and:

$$
\begin{aligned}
d \mu_{S} & =R T d \ln \left[\gamma_{ \pm}{ }^{v} c_{S}{ }^{v}\right]=\left(\nu_{+}+v_{-}\right) R T d \ln \left(\gamma_{ \pm} c_{S}\right) \\
& =v R T d \ln \left(\gamma_{ \pm} c_{S}\right)
\end{aligned}
$$

where:

$$
\gamma_{ \pm}^{v}=\gamma_{+}^{v_{+}} \gamma_{-}^{v_{-}}
$$

One should keep in mind that the solvent is water; by using an unsymmetrical reference system for the activities, (13) becomes:

$$
\begin{aligned}
x_{S} \nu R T d \ln \left(\gamma_{ \pm} c_{S}\right) & +x_{B} R T d \ln \left(\gamma_{B} c_{B}\right) \\
& +x_{W} R T d \ln \left(f_{W} x_{W}\right)=0
\end{aligned}
$$

Equation (15) is general for aqueous solutions containing a neutral molecule, $B$, together with an $\left(v_{+}-v_{-}\right)$electrolyte. By taking into account that $c_{S}$ and $c_{B}$ are around $10^{-2} \mathrm{~mol} \mathrm{~L}^{-1}$, one litre contains $c_{S}$ moles of salt, $c_{B}$ moles of $B$ and about 55.5 moles of water. So, $x_{S}=\alpha \approx c_{S} / 55.5$ and $x_{B}=\beta \approx c_{B} / 55.5$ and $x_{W} \approx 1$ whereas $f_{W} \approx 1$ because, in very dilute solutions, the solvent obeys Raoult's law. Finally, as experiments were made at constant $c_{B}$, equation (15) becomes:

$$
\alpha v R T d \ln \left(\gamma_{ \pm} c_{S}\right)+\beta R T d \ln \gamma_{B}+R T d \ln x_{W}=0
$$

Further approximations are still possible. First, according to the Debye Hückel law, $\gamma_{ \pm}$can be written as:

$$
\begin{aligned}
\log \gamma_{ \pm} & =-B\left|z_{+} z_{-}\right|\left[\left(\frac{1}{2}\right) \sum c_{i} z_{i}^{2}\right]^{\frac{1}{2}} \\
& =-B\left|z_{+} z_{-}\right|\left[\frac{1}{2}\left(v_{+} z_{+}^{2}+v_{-} z_{-}^{2}\right) c_{S}\right]^{\frac{1}{2}}
\end{aligned}
$$

where $B$ is a solvent- and temperature-dependent constant. Introducing for further use the $\frac{d c_{S}}{c_{S}}=\frac{d \alpha}{\alpha}$ term allows us
to write:

$$
d \ln \gamma_{ \pm}=-\frac{2.303}{2} B\left|z_{+} z_{-}\right|\left[\frac{1}{2}\left(v_{+} z_{+}^{2}+v_{-} z_{-}^{2}\right) c_{S}\right]^{\frac{1}{2}}\left(\frac{d \alpha}{\alpha}\right)
$$

In addition, $d \ln \left[x_{W}\right]=d \ln [1-\alpha-\beta] \approx \mathrm{d}[-\alpha-\beta] \approx-\mathrm{d} \alpha$ as $\beta=x_{B} \approx c_{B} / 55.5=$ constant in our experiments.
Consequently:

$$
\begin{aligned}
& \alpha v d \ln \alpha-\alpha v B\left|z_{+} z_{-}\right|\left[\frac{1}{2}\left(v_{+} z_{+}^{2}+v_{-} z_{-}^{2}\right) c_{S}\right]^{\frac{1}{2}} \times \\
& \left(\frac{2.303}{2}\right)\left(3 c_{S}\right)^{\frac{1}{2}}\left(\frac{d \alpha}{\alpha}\right)+\beta d \ln \gamma_{B}-d \alpha=0
\end{aligned}
$$

and finally, for a $1-2$ electrolyte at $25^{\circ} \mathrm{C}$, in an aqueous solution where $B=0.5$, we get:

$$
\begin{aligned}
\beta d \ln \gamma_{B} & =\left[-2+6\left(c_{S}\right)^{\frac{1}{2}}\right] d \alpha \\
& =\left[-2+6\left(c_{S}\right)^{\frac{1}{2}}\right] \alpha d \ln \alpha
\end{aligned}
$$

Equation (19) is valid for dilute solutions where $c_{B}$ is constant. It expresses the "salt effect" exerted upon the neutral molecule $B$ in solution by the salt at the concentration $\alpha$ (molar fraction). The induced variation of $\ln \left[\gamma_{B}\right]$ directly displaces the solubility limit of $B$; this constitutes the best evidence of the "salt effect".

By taking into account relations (13), (17) and (20), we can rewrite Equation (12) as:

$$
\begin{aligned}
& -d \sigma=R T \times \\
& {\left[\Gamma_{S, W} 3\left(1-2\left(c_{S}\right)^{\frac{1}{2}}\right) d \ln \alpha+\left(-2+6\left(c_{S}\right)^{\frac{1}{2}}\right)\left(\frac{c_{S}}{c_{B}}\right) \Gamma_{B, W}(d \ln \alpha)\right]}
\end{aligned}
$$

or:

$$
\begin{aligned}
& \Gamma_{\mathrm{Pb}\left(\mathrm{NO}_{3}\right)_{2}, W}=\left(1-2 c_{\mathrm{Pb}\left(\mathrm{NO}_{3}\right)_{2}} \frac{1}{2}\right)^{-1} \times \\
& {\left[-\left(\frac{1}{3 R T}\right)\left(\frac{\partial \sigma}{\partial \ln c_{\mathrm{Pb}\left(\mathrm{NO}_{3}\right)_{2}}}\right)+\left(\frac{2}{3}-2 c_{\mathrm{Pb}\left(\mathrm{NO}_{3}\right)_{2}}^{\frac{1}{2}}\right)\left(\frac{c_{\mathrm{Pb}\left(\mathrm{NO}_{3}\right)_{2}}}{c_{B}}\right) \Gamma_{B, W}\right]}
\end{aligned}
$$

\subsubsection{Determination of Benzene Surface Content in Presence of Lead Salt}

At constant $c_{s}$, and with the same approximations about water as above, (16) leads to:

$$
\alpha v R T d \ln \left(\gamma_{ \pm}\right)+\beta R T d \ln \gamma_{B} c_{B}+R T d \ln x_{W}=0
$$

So, (12) can be expressed as:

$$
-d \sigma=\Gamma_{S, W} R T d \ln \gamma_{ \pm}+\Gamma_{B, W} R T d \ln \gamma_{B} c_{B}
$$

and with $\log \gamma_{ \pm}=-B 2\left(3 c_{S}\right)^{\frac{1}{2}} \approx$ const 
relation (19) leads to:

$$
c_{B} d \ln \gamma_{B}=\left[-2+6\left(c_{S}\right)^{\frac{1}{2}}\right] d c_{S}
$$

If the approximation contained in Equations (25) and (26), namely the Debye-Hückel constant, $B$, is independent of benzene concentration, $\gamma_{ \pm}$and $\gamma_{B}$ are both constant, and Equation (24) very simply writes:

or:

$$
-d \sigma=\Gamma_{B, W} R T d \ln c_{B} \text { or } \Gamma_{B, W}=-\left(\frac{1}{R T}\right)\left(\frac{\partial \sigma}{\partial \ln c_{B}}\right)_{c_{S}, T}
$$

\section{MATERIALS AND METHODS}

\subsection{Bubble Column}

Most of the device characteristics depicted on Figure 2 were taken from the description by Wan and Tokunaga [13]. The column consists of a 2-m-high tube of Pyrex; its inner diameter is $19 \mathrm{~mm}$. Eight septum-capped sampling ports are distributed along the length of the tube with shorter intervals toward the top end. Its bottom end is a 5-mm high gas chamber, whose ceiling is made of fifth grade fritted glass plate; to maximise bubble concentrations, its diameter is greater than the column cross-section. When the column is filled with a solution, injection of Argon gas into the gas chamber below the fritted glass plate generates bubbles, which rise up across the column of liquid. The gas flow is controlled and measured with a smart DMFC metal-sealed/ultra high purity mass flow-meter and -controller, model 6256S by Brooks Instruments. As benzene and cyclohexane are rather volatile solutes, to avoid any loss of vapour at the top of the column during experiments, we added there a $15-\mathrm{cm}$-high double walled glass cooled at $6^{\circ} \mathrm{C}$ to be just below the freezing point of benzene. Finally, to get a fairly constant inner temperature in the column we coiled up a plastic and thermoregulated water-fed tube around the tube of Pyrex to set the temperature within the column at $25.0 \pm 0.1^{\circ} \mathrm{C}$.

Under the above conditions for the column, a gas flow rate of about $1.66 \times 10^{-7} \mathrm{~m}^{3} \mathrm{~s}^{-1}$ (measured under standard conditions) produced relatively high populations and fast streams of bubbles of small sizes. The diameters of bubbles were measured photographically with a DXC-107A/107AP Color Video Camera (Sony) equipped with a manual macro zoom with a $10^{1 / 2}$ " C frame and 18/180 $\mathrm{mm}$ focal length. The camera was connected to a computer for image acquisition. For scaling purpose, a 15-mm-diameter flat glass showing a micrometer was installed at the mid column section away from the wall for photographing. Photographs were taken at a fast shutter speed $(1 / 2000 \mathrm{~s})$ under the standard gas rate and registered by the Capturix VideoSpy software. The sizes of bubbles were determined, bubble after bubble, with the Jasc Paint Shop Pro7 software. For a given solution, the total surface area of 100 photographed bubbles was calculated on assuming spherical symmetry; the average bubble diameter was found to be $260 \pm 11 \mu \mathrm{m}$ and led to $\mathrm{a} \approx 0.22 \pm 0.02 \mathrm{~mm}^{2}$ as average area.

The time required to get a stable profile was tested by repeating the experiments with different solutions always at the same concentrations and varying time. We observed a concentration gradient only with very diluted solutions, in the range $10^{-5}-10^{-4} \mathrm{~mol} \mathrm{~L}{ }^{-1}$. Correctness for time and concentration was simply tested by verifying that a constant concentration was obtained at the upper septum after a certain period of time. Then, the concentration profile was determined. At steady state, $1.5 \mathrm{ml}$ samples were withdrawn with syringes through the sampling ports in descending order. Samples of benzene solution were poured into closed $20 \mathrm{~cm}^{3}$ flasks containing each $18 \mathrm{~cm}^{3}$ of a 50-50 (v/v) water-methanol mixture prior to their measurement by UV spectroscopy. Samples of cyclohexane were introduced in vapour-tight, septum-closed, $10 \mathrm{~cm}^{3}$ flasks. According to the nature of samples, the flasks were either used directly (head space method for cyclohexane) or to transfer the samples to the electrochemical device (lead ion determination).

\subsection{Analytical Methods}

\subsubsection{Hydrocarbons}

We checked the benzene content of solutions by UV spectroscopy according to the method described in [23]. But, as this method was inappropriate for cyclohexane, for this hydrocarbon we successfully used head-space gas chromatography [24].

A $5-\mathrm{cm}^{3}$ sample of aqueous hydrocarbon solution was placed in a previously washed and dried $10-\mathrm{cm}^{3}$ vapour-tight flask closed with a septum. For every sample, the flask was systematically stored for $20 \mathrm{~min}$ in a thermoregulated oven heated at $60^{\circ} \mathrm{C}$ to reach equilibrium within the flask headspace between dissolved hydrocarbon and vapour. Then, we withdrew a $1-\mathrm{cm}^{3}$ aliquot of vapour and injected it immediately in a DANI Educational type gas chromatograph equipped with a splitless injector at $250^{\circ} \mathrm{C}$ and a $300^{\circ} \mathrm{C}$ FID type detector; the vector gas was nitrogen. The oven was maintained at $100^{\circ} \mathrm{C}$. The column used for separation was a 30-m long, 0.32-mm-diameter SPB1 apolar one with a $0.25-\mu \mathrm{m}$-thick film of poly-dimethyl-siloxane as stationary phase.

After standardisation and adjustment of the method, a stock solution was made by shaking at $25^{\circ} \mathrm{C}$ for 3 weeks a known weight of cyclohexane in water; it is worth noting that dissolved quantity was ten-fold smaller than the solubility limit. Then, this solution was diluted to prepare a series of standard solutions; aliquots were treated as described above, 
and vapour was analysed by chromatography to make a calibration curve by plotting a straight line between the chromatographic peak area and the cyclohexane concentration.

For cyclohexane and benzene, repeatability was assessed and found to be about $1 \%$ by successively withdrawing 6 samples from the bubble column through the same mid-column septum.

\subsubsection{Lead lons $\left(\mathrm{Pb}^{++}\right)$}

Solutions containing either pure lead nitrate salt or lead nitrate and one among the hydrocarbons under study were taken from the bubble column through septa as previously described. Our experimental conditions, i.e. hydrocarbons far from their solubility limit, made us assume that their adsorption was unaffected by the presence of salt; we, therefore, only analysed the salt content. One should, however, note that cyclohexane and salt were simultaneously determined only in one sample. This will be detailed in the Results section to demonstrate that our hypothesis stands.

$\mathrm{Pb}^{++}$concentrations were determined by Differential Pulse Anodic Stripping Voltammetry, DPASV [25]. The electrochemical system consisted of a Hanging Mercury Drop Electrode (663 VA stand, Metrohm) connected to a voltammetric analyser (microAutolab, Eco Chemie). Current and potential were sampled with a personal computer. All potentials were referred to the $\mathrm{Ag} / \mathrm{AgCl}, 3 \mathrm{~mol} \mathrm{~L}^{-1} \mathrm{KCl}$ electrode. A platinum wire was used as a counter electrode. The quartz voltammetric cell was thermoregulated at $25.0 \pm 0.1^{\circ} \mathrm{C}$. The concentration in salt being about $10^{-5} \mathrm{~mol} \mathrm{~L}^{-1}$, the samples were systematically ten-fold diluted with a $10^{-2} \mathrm{~mol} \mathrm{~L}^{-1}$ nitric acid solution to slightly acidify the medium and prevent metal from adsorbing on cell walls. The samples were deoxygenated for $10 \mathrm{~min}$ with water-saturated nitrogen. The duration for accumulation was $120 \mathrm{~s}$ under a $-0.6 \mathrm{~V}$ potential, and the equilibration time was $10 \mathrm{~s}$. The voltammetric modulation time was $50 \mathrm{~ms}$, with $1 \mathrm{~s}$ as interval time, and the modulation amplitude was $50 \mathrm{mV}$ with a step potential of $20 \mathrm{mV}$.

\subsection{Surface Tensions and Wetting}

Surface tensions were measured with the Wilhelmy method while taking great care about cleanliness and control of temperature. The plate was always burnt prior to measurement to be sure of the quality of platinum surface. During measurements, the solutions were kept within a double-walled glass cell thermoregulated at $\pm 0.1^{\circ} \mathrm{C}$. The cell was placed in an oven thermoregulated at the same temperature at more or less $0.5^{\circ} \mathrm{C}$. Tensions were measured with a LAUDA TE1C tensiometer by taking the maximum force value, which means making the measurement at the exact time the liquid surface touched the plate while the cell was moving up. Reproducibility of measurements was carefully checked on taking account of all among the other possible factors, i.e. benzene volatility, adsorption kinetics; etc. Consequently, the error on surface tension was estimated to be $0.001 \sigma$.

To study the reciprocal wetting of mutually saturated water-hydrocarbon mixtures, in agreement with equations (1 to 4) we measured, at first, the surface tension of both phases against air, then their interfacial tension. After equilibration of both phases (see below) and resting, we pipetted $30 \mathrm{~cm}^{3}$ of the $\beta$, water-rich, denser phase, poured them in the cell and measured the surface tension, $\sigma_{\beta \alpha}$. Then, the Wilhelmy plate was totally sunk in the aqueous phase; we gently poured $30 \mathrm{~cm}^{3}$ of the $\gamma$, hydrocarbon-rich, lighter phase onto the aqueous phase, and measured the interfacial tension $\sigma_{\beta \gamma}$ by taking out the plate from $\beta$ to $\gamma$. Finally, after careful cleaning of the plate, we determined the surface tension of the $\gamma$ upper phase, $\sigma_{\gamma \alpha}$.

\subsection{Chemicals}

To get true molecular solutions of hydrocarbons, we first prepared saturated solutions by gently stirring with water just a little more than the necessary quantity of benzene [5] or cyclohexane. For benzene, the solubility determined at $25^{\circ} \mathrm{C}$ was $2.37 \times 10^{-2} \mathrm{~mol} \mathrm{~L}^{-1}$ [5]; this value agrees with literature data [26-27]. According to [28], the one for cyclohexane is $6.55 \pm 0.27 \times 10^{-4} \mathrm{~mol} \mathrm{~L}^{-1}$; this value is very close to our own estimation.

Water was purified on a milliQ device from Millipore and its surface tension was measured every day. Benzene, Normapur minimum 99.7 wt\%, was supplied by Prolabo. Cyclohexane Chromanorm $99.7 \mathrm{wt} \%$ for HPLC and lead nitrate, pro analysi, were supplied by Merck. These compounds were used with no further purification. The surfactant used to standardise the column was dodecylbenzenesulfonic acid, sodium salt, technical 88\% from ACROS Organics; considering its strong adsorption ability made us use it with no additional purification.

Under our experimental conditions, all the verifications we made demonstrated the lack of complexation for $\mathrm{Pb}^{++}$ ions [5-6].

\section{RESULTS AND DISCUSSION}

\subsection{Wetting}

Table 1 lists the interfacial tensions measured by tensiometry at 25 and $30^{\circ} \mathrm{C}$ in saturated diphasic mixtures of waterbenzene as well as in water-cyclohexane; $\alpha$ means vapour, $\beta$ denotes aqueous phase, $\gamma$ refers to oily phase and $\hat{\gamma}$ is the angle defined above.

One should underline that all measurements were made on the same day and on same diphasic samples. Moreover, the errors made about the benzene system (Table 1) were 
TABLE 1

Interfacial tensions in saturated diphasic mixtures of water - benzene and water - cyclohexane.

\begin{tabular}{|c|c|c|c|c|c|c|c|c|}
\hline $\begin{array}{c}\mathrm{T} \\
{ }^{\circ} \mathrm{C}\end{array}$ & $\begin{array}{c}\sigma_{\text {water }} \\
/ \mathrm{mNm}^{-1}\end{array}$ & $\begin{array}{c}\sigma_{\beta \alpha} \\
/ \mathrm{mNm}^{-1}\end{array}$ & $\begin{array}{c}\sigma_{\beta \gamma} \\
/ \mathrm{mNm}^{-1}\end{array}$ & $\begin{array}{c}\sigma_{\gamma \alpha} \\
/ \mathrm{mNm}^{-1}\end{array}$ & $\begin{array}{c}\Sigma= \\
\sigma_{\beta \gamma+} \sigma_{\gamma \alpha}\end{array}$ & M & $\hat{\gamma}^{\circ}$ & $\begin{array}{c}\text { Wetting Oily phase } \\
\text { on aqueous phase }\end{array}$ \\
\hline \multicolumn{9}{|c|}{ Benzene/water system } \\
\hline 25 & 71.90 & $70.49 \pm 0.07$ & $32.10 \pm 0.03$ & $27.96 \pm 0.03$ & $60.06 \pm 0.06$ & $1.751 \pm 0.004$ & 0 & Complete \\
\hline 30 & 71.18 & $69.70 \pm 0.07$ & $31.18 \pm 0.03$ & $27.72 \pm 0.03$ & $58.90 \pm 0.06$ & $1.803 \pm 0.004$ & 0 & Complete \\
\hline \multicolumn{9}{|c|}{ Cyclohexane/water system } \\
\hline 25 & 71.90 & $71.51 \pm 0.64$ & $46.48 \pm 0.10$ & $24.29 \pm 0.20$ & $70.77 \pm 0.30$ & $1.051 \pm 0.010$ & 0 & Just complete \\
\hline 30 & 71.18 & $71.15 \pm 0.21$ & $47.39 \pm 0.80$ & $23.85 \pm 0.11$ & $71.24 \pm 0.91$ & $0.994 \pm 0.021$ & $\begin{array}{c}6.2 \\
(0-13.3)\end{array}$ & Partial \\
\hline
\end{tabular}

estimated from a repeatability assessment conducted only on the aqueous phase. We should have done the same for the other interfaces, but it was too difficult; so, the error values about these data are likely slightly biased. But the conclusion about wetting is obviously valid.

The errors about the cyclohexane system were calculated from reproducibility estimation since the measurements had been made on the same day for each set of $\sigma\left(\sigma_{\beta \alpha}, \sigma_{\beta \gamma}, \sigma_{\gamma \alpha}\right)$ and repeated several times on different samples and days. However, one should note that the lack of systematic check of their concentrations contrarily to what was done for aqueous solutions of benzene may explain the larger dispersion of data that prevents us from concluding whether wetting is just complete, or slightly above, or slightly below; we can only say that wetting is very close to complete. In both cases, the oil-rich phase spreads totally, or nearly totally, onto the water-rich phase; it occurs more easily with the benzene system because of the greater similarity between the concentrations of both phases. It is worth noting that the difference in wetting between systems results from the outstanding difference between the values of interfacial tension Indeed, $\sigma_{\beta \gamma}$ was always found to be lower with benzene, likely because it vanishes in the close vicinity of a critical point where both concentrations are nearly alike [29-34]. In this study, since we were very far from a critical point, the system with closer equilibrium phases had, however, the smaller interfacial tension.

It is worth analysing the consequences for adsorption as done in Section 1.1. Let us consider a constant temperature; moving from dilute solutions to saturation shows that, near saturation, adsorption value is more and more affected by demixing. A surface enrichment concomitant with that of the forming new phase can be assumed from nucleation theories despite the layer thickness-dependency of adsorption value. Consequently, at identical concentrations in solution, close to the saturation concentration of cyclohexane in water, its adsorption should be greater than the benzene one. We will see later whether experiments confirmed this hypothesis.

\subsection{Adsorptions}

To test the bubble column, we used SDBS and compared the results with those issued from surface tension measurements. SDBS is very surface-active, even in very dilute solutions where the use of the bubble column is relevant. At $25^{\circ} \mathrm{C}$ and concentrations for solutions in the range $10^{-5}-2 \times 10^{-4}$ mol $\mathrm{L}^{-1}$, the surface tension varies linearly with $C$, the solution concentration, between 70 and $50 \mathrm{mN} \mathrm{m}^{-1}$. Then, the adsorption provided by the Langmuir isotherm formula can be simply expressed as:

$$
\Gamma=K_{\gamma} C
$$

where $\mathrm{K}_{\gamma}=4.703 \times 10^{-5} \mathrm{~m}$.

The experiment made at $\mathrm{C}_{0}=1.99 \times 10^{-5}$ mol L-1 with the bubble column under conditions already described (namely, $f=1.666 \times 10^{-7} \mathrm{~m}^{3} \mathrm{~s}^{-1}, D=6.10 \times 10^{-4} \mathrm{~m} \mathrm{~s}^{-1}$, $A=3.14 \times 10^{-4} \mathrm{~m}^{2}$ ) led to $\mathbf{J}=0.850 \mathrm{~m}^{-1}$, and from Equation (5) to $\mathbf{K}=4.780 \times 10^{-5} \mathrm{~m}$, which is very close to the value found for $K_{\gamma}$.

The excellent agreement between these data mainly results from the good adsorbability and separability of the surfactant that led to a high $\mathbf{J}$ and permitted its very precise determination $(0.35 \%)$. In the other experiments, the values found for $\mathbf{J}$ were far much lower.

The adsorption constants of hydrocarbons in water, $K_{C}$ and $K_{B}$ for cyclohexane and benzene, respectively, were determined at $25^{\circ} \mathrm{C}$ under various experimental conditions and for different concentrations of hydrocarbon. On the other hand, for the determination, at $25^{\circ} \mathrm{C}$, of $K_{P b, C}$ and $K_{P b, B}$ in the case of lead nitrate in aqueous solutions of cyclohexane and benzene, respectively, the only factor to be varied was the lead salt concentration. Even though the concentration in hydrocarbon was kept unchanged, it needed to meet two unavoidable constraints:

- produce a surface content high enough to induce metallic salt adsorption; 
- be sufficiently low to permit the observation of a concentration gradient in the bubble column.

As previously underlined, such an observation is impossible when the mean concentrations in the column are too high. The narrowness of the operating window made us carry out our investigations in presence of salt at a single hydrocarbon concentration.

Table 2 introduces our results, and the experimental data at the origin of these values are reported in Figures 3 to 6 as bubble column-normalised concentration profiles $\left(C(z) / C_{0}\right)_{i}$ with $i$ indexing the species under study. The profiles displayed on Figures 3 and 4 are those of cyclohexane and benzene in solutions, respectively; Figures 5 and 6 show those of lead salt in presence of cyclohexane and benzene, respectively. At similar $a$ and $f$ values, such a plot allows one to check the consistency of data with the hypothesis of Equation (5), namely the adsorption proportionality to bulk concentration (constant $\mathbf{K}$ ). Equation (8) then leads to the $\mathbf{J}$ parameter, which was exploited through Equation (7) to get $K_{i}$ where $i$ was $C$ or $B$, with or without a co-adsorbate. In Equation (7), $A$ and $f$ were given the following values $A=3.14 \times 10^{-4} \mathrm{~m}^{2}$, $f=1.666 \times 10^{7} \mathrm{~m}^{3} \mathrm{~s}^{-1}$. After determination, the $a$ parameter was considered to be $a \approx 0.22 \mathrm{~mm}^{2}$ with a dispersion of $12 \%$ for every experimental run. Figures. 2 to 5 also display the lines representing the data issued from Equation (7) and highlight a quite good agreement between lines and experimental points. Reproducibility on $\mathbf{J}$ was assessed to be $10 \%$ through six complete runs of experiments made at the same concentration of benzene, but on different days. A numerical analysis of the confidence degree on $\mathbf{J}$ according to the numerical procedure at the origin of its calculation gave also $10 \%$.

As $\mathbf{J}$ was assumed to be constant, it was calculated numerically from Equation (8) under the linearised form where the slope is exactly $J$ :

$$
\ln \left(\frac{C(z)}{C_{0}}\right)=\mathbf{J} z+\ln \left[\frac{H \mathbf{J}}{\exp (H \mathbf{J})-1}\right]
$$

All the series of data corresponding to different runs on the same system can be drawn on the same straight line; So, J was determined with a regression method to minimise the sum of square differences between the experimental values of:

$$
\ln \left[\frac{C(z)}{C_{0}}\right]
$$

To estimate the confidence degree on $\mathbf{J}$ from the numerical determination, we used a Gauss-Newton method [35]. For SDBS and benzene, $\Delta \mathbf{J}$ was $0.003(\Delta \mathbf{J} / \mathbf{J}=0.35 \%$ and $\Delta \mathbf{J} / \mathbf{J}$ $=3.5 \%$, respectively); for cyclohexane and lead nitrate in any hydrocarbon mixture $\Delta \mathbf{J} / \mathbf{J}=4.5 \%$.

TABLE 2

Adsorption constants, separation factors and corresponding adsorptions for sodium dodecylbenzenesulfonate (SDBS),

\begin{tabular}{|c|c|c|c|c|c|c|}
\hline $\begin{array}{l}\text { Species } i \\
\text { symbols }\end{array}$ & $K_{i} / m$ & $\mathrm{~J}_{\mathrm{i}} / \mathrm{m}^{3}$ & $\mathrm{n}$ & $\begin{array}{l}\Gamma_{i} \text { or } \Delta \Gamma_{i} / \mathrm{mol} \mathrm{m}^{-2} \\
C_{i} \text { or } \Delta C_{i} / \mathrm{mol} \mathrm{L}^{-1}\end{array}$ & $\begin{array}{l}\Gamma_{H} / \mathrm{mol} \mathrm{m}^{-2} \\
C_{H} / \mathrm{mol} \mathrm{L}^{-1}\end{array}$ & $\begin{array}{l}\Delta \Gamma_{P b, H} / \mathrm{mol} \mathrm{m}^{-2} \\
\Delta \mathrm{C}_{P b, H} / \mathrm{mol} \mathrm{L}^{-1}\end{array}$ \\
\hline SDBS & $(4.78 \pm 0.55) \times 10^{-5}$ & $\begin{array}{c}0.850 \\
\pm 0.003 \\
\end{array}$ & (1) & $\begin{array}{l}9.35 \times 10^{-7} \\
1.99 \times 10^{-5}\end{array}$ & & \\
\hline $\mathrm{C}$ & $(3.73 \pm 0.97) \times 10^{-6}$ & $\begin{array}{r}0.09 \\
\pm 0.004\end{array}$ & $(3+1)$ & $\begin{array}{l}(0.09-1.49) \times 10^{-7} \\
(2.47-40.0) \times 10^{-6}\end{array}$ & & \\
\hline B & $(1.05 \pm 0.26) \times 10^{-6}$ & $\begin{array}{c}0.023 \\
\pm 0.0008\end{array}$ & (6) & $\begin{array}{c}(3.15-6.30) \times 10^{-7} \\
(3.0-6.0) \times 10^{-4}\end{array}$ & & \\
\hline $\mathrm{Pb}, \mathrm{C}$ & $(3.27 \pm 0.85) \times 10^{-7}$ & $\begin{array}{c}0.0079 \\
\pm 0.0004\end{array}$ & $\begin{array}{c}(2) \\
(+1 \text { at } \\
\left.C_{P b, C}=2.0 \times 10^{-5}\right)\end{array}$ & & $\begin{array}{l}1.49 \times 10^{-7} \\
4.0 \times 10^{-5}\end{array}$ & $\begin{array}{l}(3.13-4.41) \times 10^{-9} \\
(0.96-1.35) \times 10^{-5}\end{array}$ \\
\hline $\mathrm{Pb}, \mathrm{B}$ & $(8.29 \pm 2.16) \times 10^{-7}$ & $\begin{array}{c}0.02 \\
\pm 0.0007\end{array}$ & (2) & & $\begin{array}{l}5.31 \times 10^{-7} \\
5.0 \times 10^{-4}\end{array}$ & $\begin{array}{c}(12.4-19.5) \times 10^{-9} \\
(1.5-2.35) \times 10^{-5}\end{array}$ \\
\hline
\end{tabular}
cyclohexane (C), benzene (B), and lead salt in presence of one of these hydrocarbons. $n$ is the number of experiments 


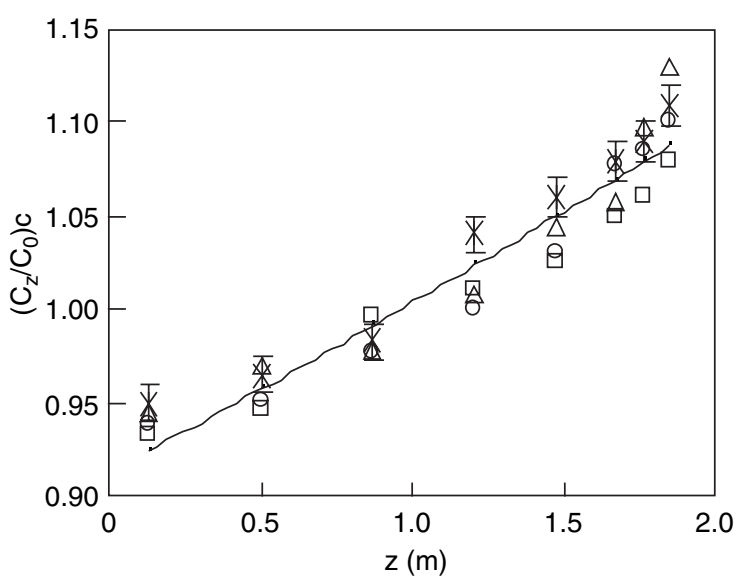

Figure 3

Cyclohexane relative concentration profile $\left(C_{z} / C_{0}\right)_{C}$ along the bubble column. $C_{z}$ is the cyclohexane molarity at $z$ altitude and steady state, during the passing of bubbles. $C_{0}$ is the initial homogeneous cyclohexane concentration. The solid line represents the four series of data according to equation (29).

๖: $\left(C_{0}\right)_{C}=2.47 \times 10^{-6} \mathrm{~mol} \mathrm{~L}^{-1} ; \quad$ O: $\left(\mathrm{C}_{0}\right)_{\mathrm{C}}=5 \times 10^{-6} \mathrm{~mol} \mathrm{~L}^{-1}$; $\Delta:\left(C_{0}\right)_{C}=8.6 \times 10^{-6} \mathrm{~mol} \mathrm{~L}^{-1} ; \times:\left(C_{0}\right)_{C}=4 \times 10^{-5} \mathrm{~mol} \mathrm{~L}^{-1}$.

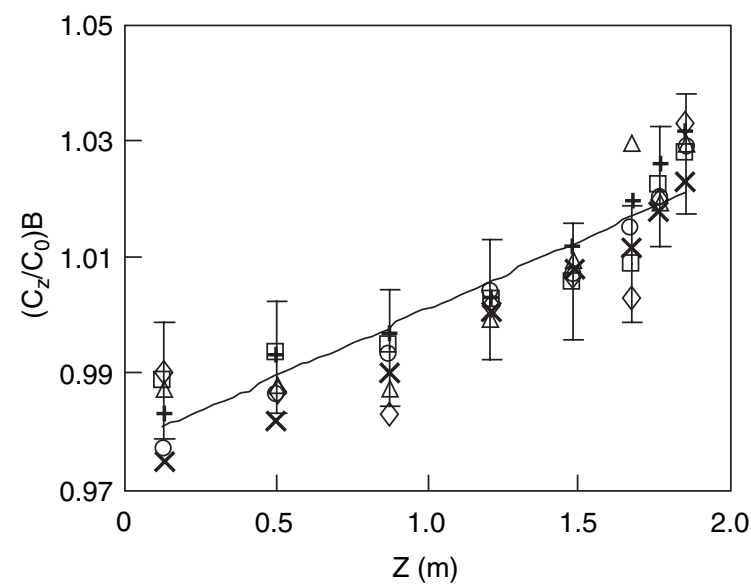

Figure 4

Benzene relative concentration profile $\left(\mathrm{C}_{\mathrm{Z}} / \mathrm{C}_{0}\right)_{\mathrm{B}}$ in the bubble column. $\mathrm{C}_{\mathrm{z}}$ is the benzene molarity at $z$ altitude and steady state during the passing of bubbles. $\mathrm{C}_{0}$ is the initial benzene concentration within a homogeneous solution. The solid line represents the six series of data according to Equation (29).

○: $\left(\mathrm{C}_{0}\right)_{B}=3 \times 10^{-4} \mathrm{~mol} \mathrm{~L}^{-1} ; \quad \square:\left(\mathrm{C}_{0}\right)_{B}=3.5 \times 10^{-4} \mathrm{~mol} \mathrm{~L}^{-1}$;

$\Delta:\left(\mathrm{C}_{0}\right)_{B}=4 \times 10^{-4} \mathrm{~mol} \mathrm{~L}^{-1} ; \quad$ O: $\left(\mathrm{C}_{0}\right)_{B}=5 \times 10^{-4} \mathrm{~mol} \mathrm{~L}^{-1}$;

$\mathrm{x}:\left(\mathrm{C}_{0}\right)_{B}=5.5 \times 10^{-4} \mathrm{~mol} \mathrm{~L}^{-1} ;+:\left(\mathrm{C}_{0}\right)_{B}=6 \times 10^{-4} \mathrm{~mol} \mathrm{~L}^{-1}$.
Table 2 also gives $\mathrm{J}_{\mathrm{i}}$ and the corresponding $K_{i}$ values with estimated uncertainties.

$$
\frac{\Delta \mathbf{K}}{\mathbf{K}}=\frac{\Delta a}{a}+\frac{\Delta D}{D}+\frac{\Delta \mathbf{J}}{\mathbf{J}}=0.12+0.10+\frac{\Delta \mathbf{J}}{\mathbf{J}}=0.22+\frac{\Delta \mathbf{J}}{\mathbf{J}}
$$

$(\Delta \mathbf{K} / \mathbf{K})$ falls between 0.255 and 0.265 with our systems. According to Equation (30), the errors on $A$ and $f$ being negligible and the other ones being estimated as previously explained, because of the uncertainties on $\mathbf{K}$ values, only the marked effects upon adsorption are worth being discussed.

The discussion about $K_{C}$ and $K_{B}$ values in the case of only two components is much easier than under other conditions. Indeed, their determination on concentration ranges centred at $10^{-5}$ and $5 \times 10^{-4} \mathrm{~mol} \mathrm{~L}^{-1}$ for cyclohexane and benzene, respectively, ensures the validity of the values found for $\mathbf{K}$ because it corresponds to a true linear behaviour at the start of adsorption isotherms as assumed in Equation (5). Though the bulk concentrations were closer to the solubility limit of cyclohexane $\left((6.55 \pm 0.27) \times 10^{-4} \mathrm{~mol} \mathrm{~L}^{1}\right)$ than to that of benzene $\left((2.37 \pm 0.02) \times 10^{-2} \mathrm{~mol} \mathrm{~L}^{-1}\right)$, they were very far from them. Finally, as $K_{C}$ is almost four times $K_{B}$, it means that, in very dilute solutions and far from the influence of solubility limits, cyclohexane adsorbs more than benzene. However, the difference between $K$ values has likely the same origin as the difference in solubility limits: the (slightly) greater affinity of water for benzene compared to cyclohexane results from possible interactions between the electron-rich population of the $\pi$ system and hydrogen atoms of water [36] responsible for an enhancement of benzene solubility. In dilute solutions, solute-solvent interactions are known to play a pre-eminent role.

Our measurements of lead nitrate adsorption were made at a single bulk initial concentration for each hydrocarbon: namely $4 \times 10^{-5}$ and $5 \times 10^{-4} \mathrm{~mol} \mathrm{~L}^{-1}$ for cyclohexane and benzene, respectively. These bulk concentrations correspond to adsorptions of $1.57 \times 10^{-7}$ and $5.31 \times 10^{-7} \mathrm{~mol} \mathrm{~m}^{-2}$, respectively, imposed by experimental constraints and calculated by using the $K_{C}$ and $K_{B}$ values determined in hydrocarbon solutions. However, a determination of $K_{C}$ made in presence of lead nitrate (Table 1) gave the same value and evidenced the correctness of our estimated values. Thus:

- very far from hydrocarbon solubility limits, the salt effect on adsorption is quite negligible,

- in these composite systems, the establishment of parallel concentration profiles, both governed by the same rules (Equations 5 to 9) seems unaffected by the bubbleinduced perturbations.

The more general conclusion drawn from Table 2 data about lead salt adsorption is that this process occurs even with water-dissolved traces of hydrocarbon. Lead adsorption constants are about ten-fold less than that of cyclohexane and alike that of benzene:

$$
\frac{K_{P b, C}}{K_{C}}=0.084 \text { and } \frac{K_{P b, B}}{K_{B}}=0.86
$$

The adsorption ratios between a hydrocarbon and a metallic salt for these dilute solutions can be calculated from the ratios of adsorption constants as defined by Equation (5). 


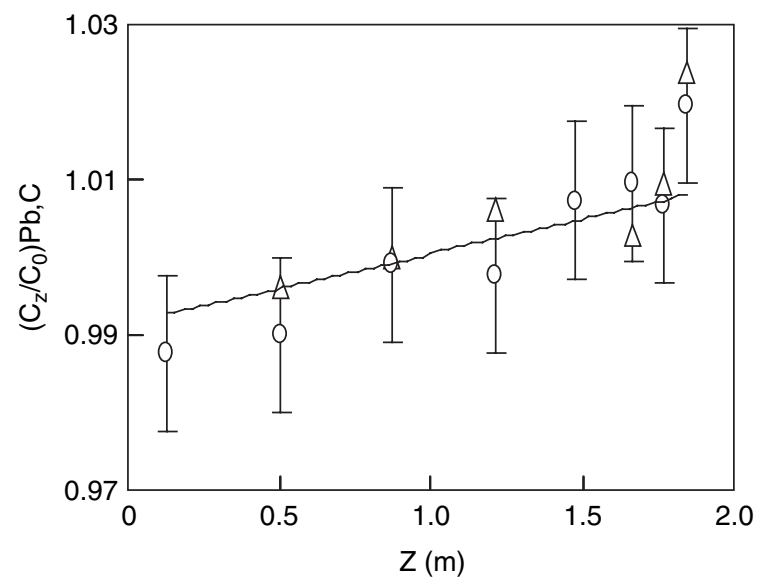

Figure 5

Lead nitrate relative concentration profile $\left(C_{z} / C_{0}\right)_{P b, C}$ in the bubble column in presence of cyclohexane. $\left(C_{z}\right)_{P b, C}$ is the lead nitrate molarity at $z$ altitude and at steady state during the passing of bubbles. $\left(C_{0}\right)_{P b, C}$ and $\left(C_{0}\right)_{C}$ are respectively the initial homogeneous salt and (constant) cyclohexane concentrations. The solid line represents the series of data according to Equation (29).

$\Delta:\left(C_{0}\right)_{P b, C}=9.6 \times 10^{-6} \mathrm{~mol} \mathrm{~L}^{-1} ;$ ○: $\left(C_{0}\right)_{P b, C}=1.35 \times 10^{-5} \mathrm{~mol} \mathrm{~L}^{-1}$; $\left(C_{0}\right)_{C}=4 \times 10^{-5} \mathrm{~mol} \mathrm{~L}^{-1}$

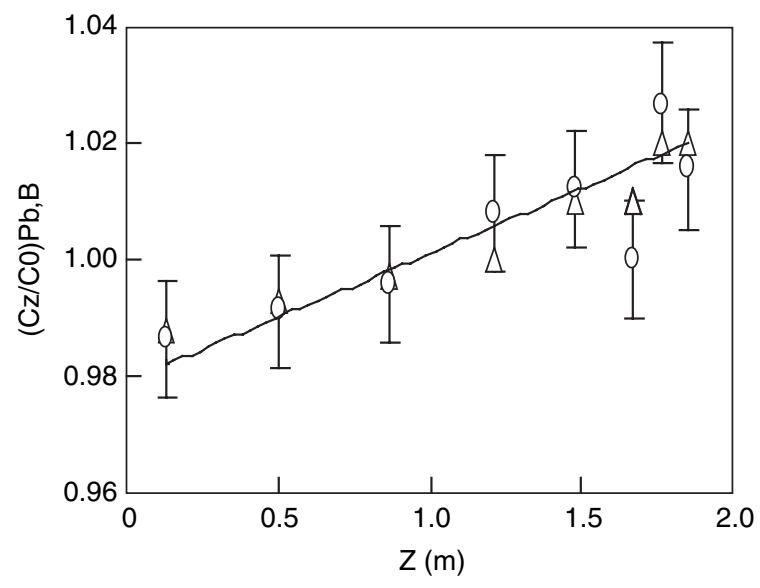

Figure 6

Lead nitrate relative concentration profile $\left(C_{z} / C_{0}\right)_{P b, B}$ in the bubble column in presence of benzene. $\left(C_{z}\right)_{P b}$ is the lead nitrate molarity at $z$ altitude and steady state during the passing of bubbles. $\left(C_{0}\right)_{P b}$ and $\left(C_{0}\right)_{B}$ are respectively the initial homogeneous salt and (constant) benzene concentrations. The solid line represents the double series of data according to Equation (29). $\Delta:\left(C_{0}\right)_{P b, B}=2.5 \times 10^{-5} \mathrm{~mol} \mathrm{~L}^{-1} ;$ O: $\left(C_{0}\right)_{P b, B}=2.35 \times 10^{-5} \mathrm{~mol} \mathrm{~L}^{-1}$; $\left(C_{0}\right)_{B}=5 \times 10^{-4} \mathrm{~mol} \mathrm{~L}^{-1}$.
Assuming that $K_{P b, H}$ is independent of the $H$ concentrations, which has not yet been experimentally proven, leads to:

and:

$$
\frac{\Gamma_{P b, C}}{\Gamma_{C}}=0.084 \frac{C_{P b, C}}{C_{C}}
$$

$$
\frac{\Gamma_{P b, B}}{\Gamma_{B}}=0.86 \frac{C_{P b, B}}{C_{B}}
$$

Equations (31)-(33) can then be read as follows: for the same bulk concentration ratio $\left(\mathrm{C}_{\mathrm{Pb}, \mathrm{H}} / \mathrm{C}_{\mathrm{H}}\right)$, the adsorption ratio $\left(\Gamma_{\mathrm{Pb}, \mathrm{H}} / \Gamma_{\mathrm{H}}\right)$ for benzene is ten-fold that of cyclohexane; thus, one mole of benzene attracts ten times more moles of lead salt than cyclohexane does.

Figure 7 summarises Table 2 under the form of adsorption isotherms for pure benzene, cyclohexane and lead salt in presence of one of these hydrocarbons. The resulting graph highlights the concentration ranges where measurements were possible and facilitates a visual comparison of the different values of $\mathbf{K}$ corresponding to the slopes of straight lines constituting the isotherms. Figures 8 and 9 gather both types of data presented uniquely versus hydrocarbon concentrations, and allow one to visually compare both systems. Figure 8 compares not only the adsorption of the pure hydrocarbons, but also the salt one at constant salt concentration versus the corresponding concentration of hydrocarbon. These isotherms are simply the linear interpolation between zero and a point calculated from Table 2 for a salt content equal to the highest concentration of hydrocarbon used in our experiments. Under these conditions and as expected from equations (32) and (33), salt adsorption is stronger in the presence of benzene than in the presence of cyclohexane. The linear interpolation is rather hard, but data recently obtained within our laboratory on another system showed the relevance of this hypothesis [37].

Figure 9 was obtained by drawing salt adsorption isotherms for the same concentrations of salt, but different ones for hydrocarbons (see the experimental constraints already mentioned, which restricted the ranges of values given in Table 2). At low hydrocarbon content, which corresponds to the case where the comparison is realistic because of the solubility limit of cyclohexane, salt adsorption for identical values of $C_{H}$ (along a vertical line) is reduced when cyclohexane is replaced by benzene; this observation is puzzling because it disagrees with the above analysis. This discrepancy comes from either a wrong building of the salt isotherms, or the behaviour observed here means that that adsorption is more affected by the $C_{H} / C_{\text {salt }}$ ratio than by the nature of the hydrocarbon under study. One should note that Figure 9 allows such a comparison only under certain conditions; in the case of cyclohexane, because of its solubility limit, the ratio is far much higher.

Finally, for solutions of pure benzene, Figure 10 highlights a marked decrease of surface tension near the solubility limit responsible for a strong enhancement of adsorption as expected from wetting data. Figure 11 illustrates the changes in surface tension induced by the presence of lead nitrate in the solutions of benzene, and Figure 12 gives the corresponding salt adsorption values calculated as reported in Section 1.3. As already observed in dilute solutions, the adsorption of salt is dependent on benzene and salt concentrations. 


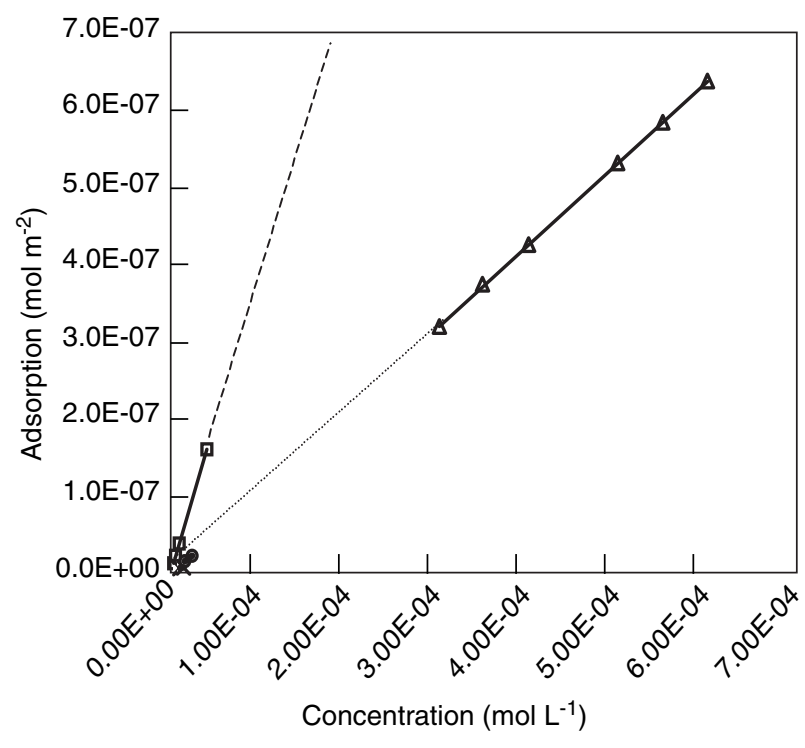

Figure 7

Linear adsorption isotherms according to Equation 5 and Table 2, at $25^{\circ} \mathrm{C}$ (adsorption of species $i$, versus $i$ bulk concentration). $\checkmark$ Cyclohexane $\Delta$ Benzene

Dots correspond to experimentally used hydrocarbons or salt concentrations.

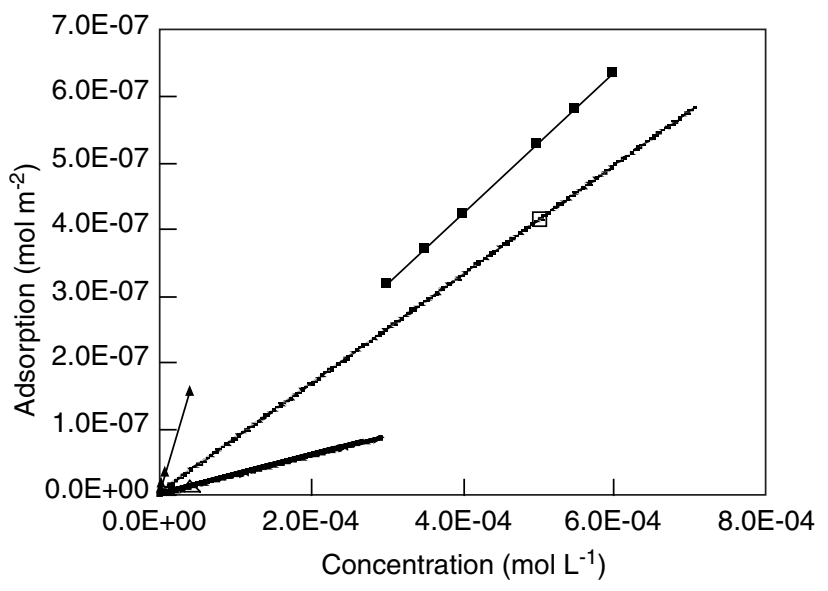

Figure 8

Linear adsorption isotherms versus the corresponding hydrocarbon concentration only.

ه. Cyclohexane adsorption versus cyclohexane concentration.

$\Delta$. Lead nitrate adsorption in presence of cyclohexane versus cyclohexane concentration for $C_{\text {salt }}=4 \times 10^{-5} \mathrm{M}\left(=C_{C}\right.$ during the experiments with both solutes) all along the straight line (there is a single experimental point on the line, except zero).

- Benzene adsorption versus benzene concentration.

๑. Lead nitrate adsorption in presence of benzene versus benzene concentration for $C_{\text {salt }}=5 \times 10^{-4} \mathrm{M}\left(=\mathrm{C}_{\mathrm{B}}\right.$ during the experiments with both solutes) all along the straight line (there is a single experimental point on the line, except zero).

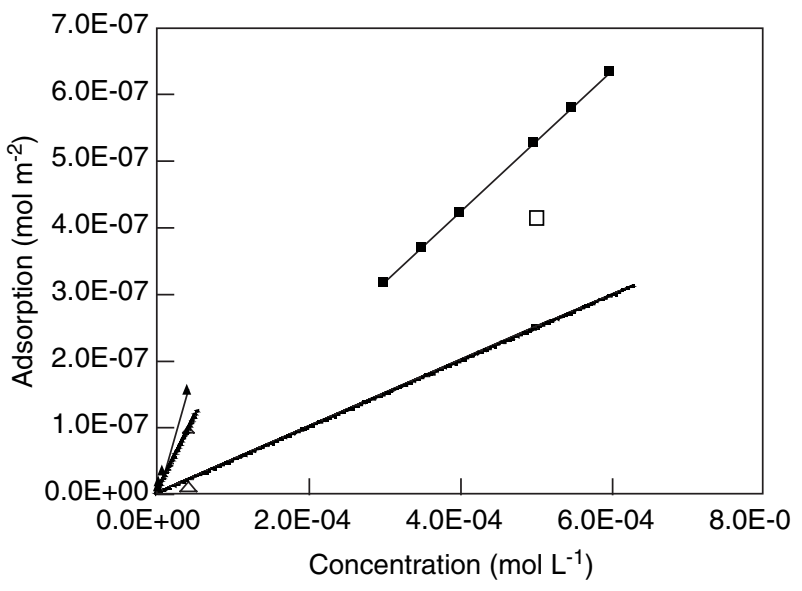

Figure 9

Linear adsorption isotherms versus the corresponding hydrocarbon concentration only. Here, the salt concentration for both salt adsorption isotherms is alike in presence of either cyclohexane or benzene.

\. Cyclohexane adsorption versus cyclohexane concentration

I. Benzene adsorption versus benzene concentration

$\square, \Delta$. Same data as in Figure 8. Along the vertical line passing by these dots, $C_{\text {salt }}=C_{H}$.

$\Delta$. Lead nitrate adsorption in presence of cyclohexane versus cyclohexane concentration for $C_{\text {salt }}=3 \times 10^{-4} \mathrm{M}$.

- Lead nitrate adsorption in presence of benzene versus benzene concentration for $C_{\text {salt }}=3 \times 10^{-4} \mathrm{M}$.

Along the vertical lines passing by the two last dots, $C_{\text {salt }} / C_{H}$ ratios are different. The resulting salt adsorption isotherms behave differently from those depicted on Figure 8 .

The influence of organic molecules on ionic adsorption can be crudely explained from the premises of Samaras and Onsager's theoretical treatment. As already described in the literature (see for example, reference [38]) or observed here on lead nitrate solutions, electrolyte solutions in water show the depletion of electrolyte from the surface. To explain this phenomenon Onsager and Samaras [39], themselves inspired by Wagner [40] (see also [41]) begun their model by using the classical method of electrostatic images. This method states that each charge, $q$, in a medium, $A$, of dielectric constant, $\varepsilon$, situated at the distance, $z$, from the plane boundary between $A$ and a second medium, $B$, of dielectric constant, $\varepsilon^{\prime}$, (Fig. 13) can be considered as being under the influence of an image charge situated in $B$ at the position $-z$ and whose value $q$ ' is:

$$
q^{\prime}=\left[\left(\varepsilon-\varepsilon^{\prime}\right) /\left(\varepsilon+\varepsilon^{\prime}\right)\right] q
$$

Let water and air be respectively $A$ and $B$, it leads to $\varepsilon=78$ and $\varepsilon^{\prime}=1$, and thus:

$$
q^{\prime} \approx q
$$




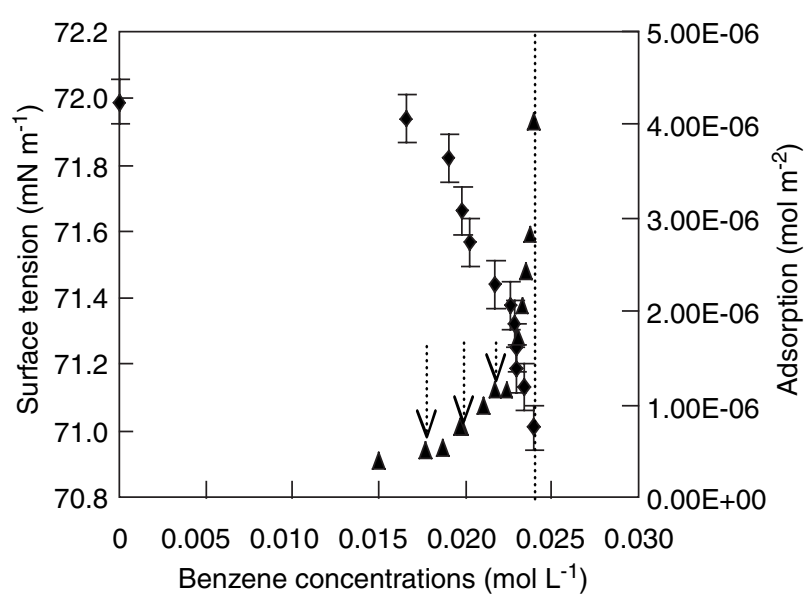

Figure 10

Surface tension $(\bullet)$ of solutions of benzene in pure water at $25^{\circ} \mathrm{C}$, and adsorption $(\boldsymbol{\Lambda})$ at the free surface versus the molarity in benzene. The dashed line shows the solubility limit. The arrows show the composition of the "solvents" used later for solutions of lead nitrate.

As $q$ ' and $q$ signs are alike, the interaction force is repulsive and leads to ion depletion near the surface.

Let us, now, suppose that $q$ enters a surface layer where $\varepsilon=2$, i.e. about the dielectric constant of organic solvents like cyclohexane or benzene.

Then:

$$
q^{\prime} \approx q / 3
$$

The coulombic force between $q$ and $q$ ' is still repulsive (both signs are still alike), but strongly reduced. Consequently, a state of the system where $q$ is embedded in the organic layer is less energetic and must be observed.
Then, as charge $q$ and the charges of similar sign will be roughly situated closer to the surface, the number measuring their adsorption will become larger. It is clear that other effects such as the screening effect on $q$ of the other charges present in the solution or the dispersion forces due to species polarisability as underlined in reference [42] have to be taken into account.

Our experiments obviously showed a reduction of ion depletion in presence of benzene and cyclohexane; but, it is worth noting that, despite their almost identical dielectric constants, salt depletion is different in both cases. Either the simple argument of image forces proposed above needs to be used in a more elaborated way, or it is insufficient to explain the experimental observations as suggested by references [41] and [42].

However, it is worth keeping in mind that a thick and dense organic molecular layer at the surface will favour ionic adsorption; it will be all the more true as its properties will be closer to those of an organic liquid. This finding explains why, on Figure 8, ion adsorption and that of the organic solvent are concomitantly increasing.

\subsection{Environmental Impact}

To discuss the impact of this physical behaviour on the environment, one should, first, take into account the order of magnitude of concentrations. In this study, the solute contents were rather close to those usually found in the environment: for example, lead was at $10^{-5} \mathrm{~mol} \mathrm{~L}^{-1}$, i.e. $2.07 \mathrm{mg} \mathrm{L}^{-1}$, i.e. just four times the upper limit found in natural fresh waters $\left(0.5 \mathrm{mg} \mathrm{L}^{-1}\right.$ in quite fresh water, $0.03 \mathrm{mg} \mathrm{L}^{-1}$ in hard water and $0.0039 \mathrm{mg} \mathrm{L}^{-1}$ as the mean value for natural water in continental North America according to [43]).
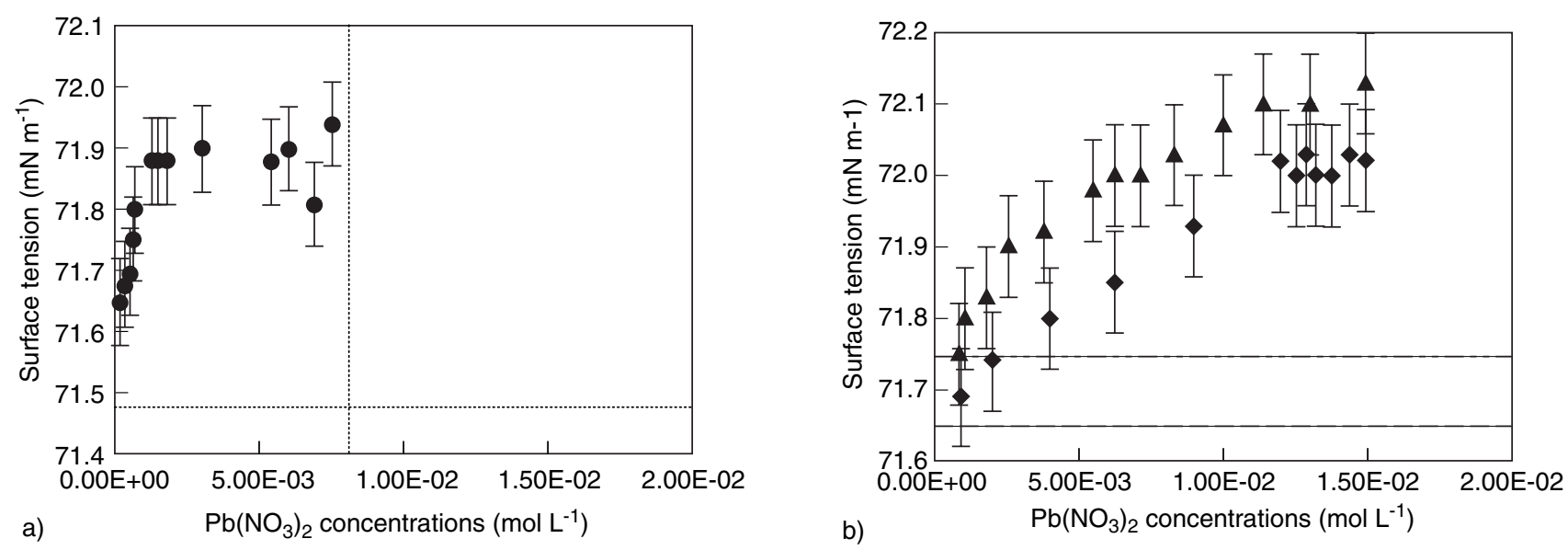

Figure 11

Surface tensions of aqueous solutions of lead nitrate versus the salt concentration in presence of dissolved benzene and at several benzene concentrations: (a) $c_{B}{ }^{1}=2.17 \times 10^{-2} \mathrm{~mol} \mathrm{~L}^{-1}=0.915 c_{O B}$ (৫); (b) $c_{B}{ }^{2}=1.97 \times 10^{-2} \mathrm{~mol} \mathrm{~L}^{-1}=0.83 c_{O B}(\bullet)$ and $c_{B}{ }^{3}=1.77 \times 10^{-2} \mathrm{~mol} \mathrm{~L}^{-1}=0.75 c_{O B}(\boldsymbol{\Delta})$.

The horizontal lines show the surface tension of the "solvent". 


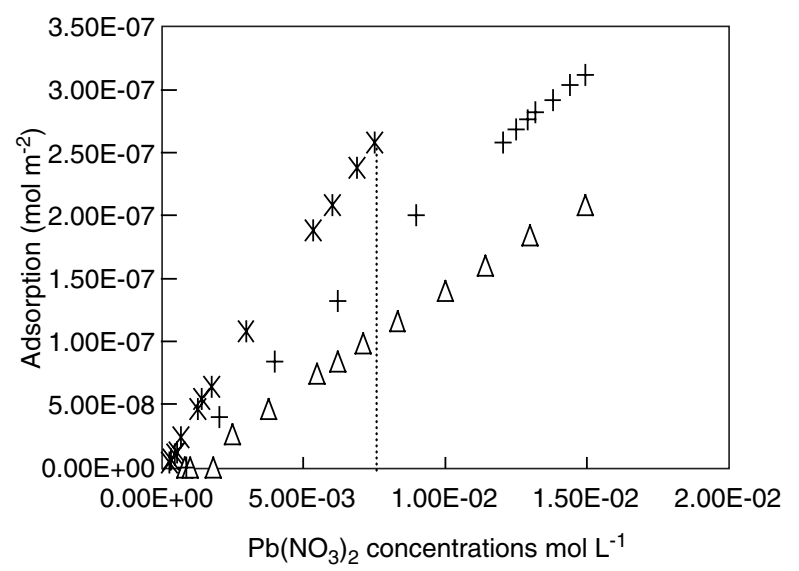

Figure 12

Adsorption isotherm of lead nitrate at $25^{\circ} \mathrm{C}$ from solutions using three different "solvents", i.e. water with three different contents of benzene:

$c_{B}{ }^{1}=0.915 c_{O B}\left({ }^{*}\right) ; c_{B}{ }^{2}=0.83 c_{O B}(+)$ and $c_{B}{ }^{3}=0.75 c_{O B}(\mathbf{\Delta})$.

The adsorption values were obtained from Equation (22) by using the surface tensions displayed on Figure 11 and the $\Gamma_{B}$ values on Figure 10. The dashed line shows the salt concentration that sets the solubility limit of benzene at concentration $c_{B}{ }^{1}$.

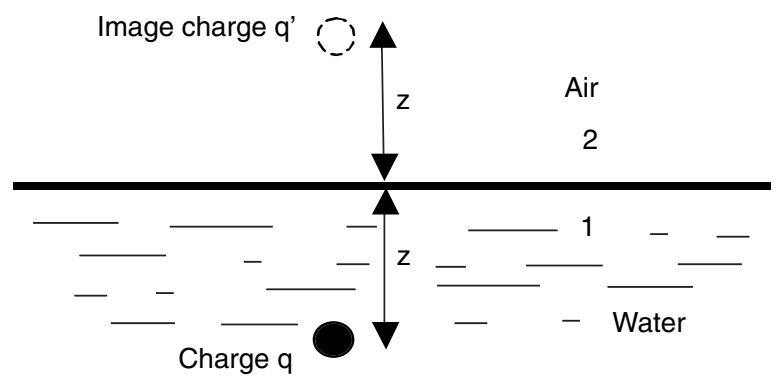

Figure 13

Scheme of the reciprocal influence between a charge $q$ and its image charge $q^{\prime}$, situated near a surface separating two media with different dielectric constants $\varepsilon_{1}$ and $\varepsilon_{2}$.
Let us now consider the role of coadsorption in bubbling and via-aerosol pollutant transfer. Bubbling leads to the formation of aerosols through the four-step process described in Figure 14:

- inclusion of air bubbles close to the water surface under the action of wind;

- bursting of bubbles giving birth to very small drops blown away by wind;

- streaming of the surface layer along the surface of the hole remaining after the bubble bursting;

- formation of a jet drop while this hole is closing.

Step 1 leads to the creation of a surface that holds an adsorption layer containing the adsorbable pollutants present in solution. The drops created in step 2 are formed from the bubble wall mainly constituted of the adsorbed layer: these droplets enriched by adsorption will later form polluted aerosols. Step 3 explains how the jet drop formed in step 4 is in turn enriched in adsorption layer components. "Tearing", which leads to the formation of "spray drops" at the wave edge or at its breaking, also contributes to aerosol formation and implies adsorption layers.

So, in ordinary environmental conditions it is paramount to consider the process of coadsorption and its associates, bubbling and via-aerosol transfer because of the significance of adsorption values shown in the present study.

Very few investigations have been focused on aqueous solutions of hydrocarbons though in industrial wastelands, water sheets polluted with hydrocarbons at trace levels and variable quantities of heavy metal salts frequently exist. Their contribution to, at least, local atmospheric pollution should be investigated. It is likely that the case of salted water sheets is worth being considered. Even though $\mathrm{NaCl}$ was missing in this study, the results reported here give some precious clues about the general behaviour of pollutants, all the more as salt is known to affect the solubility limit of hydrocarbons and, consequently, their coadsorption.

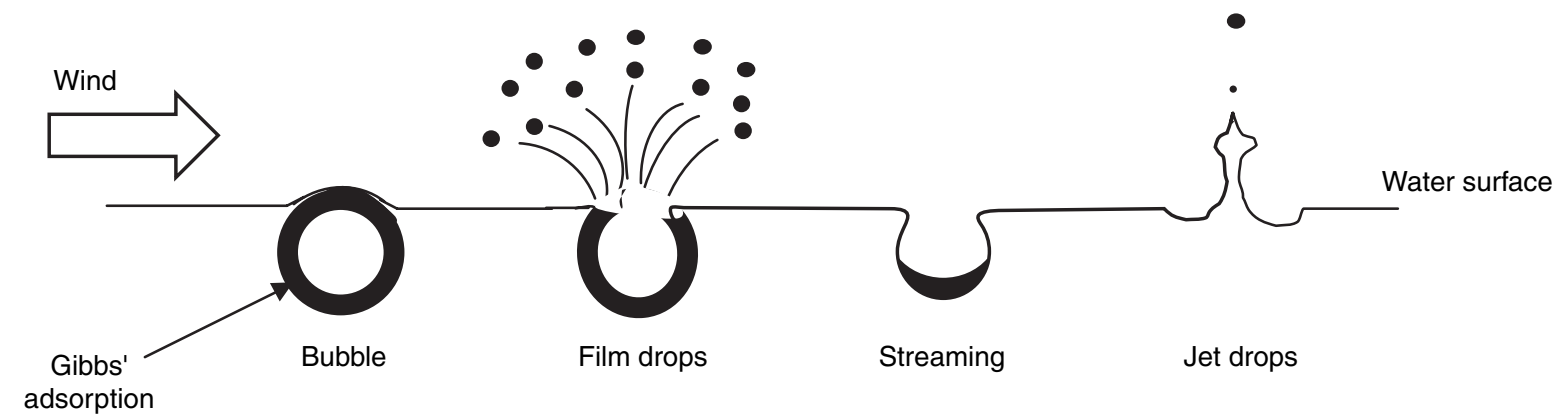

Figure 14

Scheme of bubbling mechanism. 


\section{CONCLUSION}

Despite its unavoidable experimental limits, this study clearly shows how the adsorption of two cyclic, aliphatic or aromatic hydrocarbons with the same number of carbons is affected by:

- the molecular structure;

- the solubility limit;

- the reciprocal wetting between hydrocarbon-rich phase and solvent-rich phase at the solubility limit. It gives a new insight into adsorption process by evidencing the induction of heavy metal salt coadsorption by hydrocarbon adsorption. It showed that the extent of coadsorption depends on:

- the nature and solubility limit of the hydrocarbons under study;

- hydrocarbon adsorption;

- the ratio between hydrocarbon and salt concentrations.

However, the main conclusion is that hydrocarbon adsorption is responsible for coadsorption, since no absorption of salt took place when hydrocarbon was missing. Metal could be also adsorbed as a complexed species, but we showed that it formed no complex in solution under our experimental conditions.

A part of these data was produced with the bubble column, a method scarcely used, which proved its relevance and usefulness in the study of adsorption process with surface tension-inactive systems.

Finally, the coadsorption mechanism described here may explain the passing of scarcely soluble, non adsorbable compounds from fresh water to the atmosphere. It is likely that such investigations should be relevant in the case of salted waters.

\section{REFERENCES}

1 Gros, V., Martin, D., Poisson, N., Kanakidou, M., Bonsang, B., Le Guern, F. and Demont, D. (1998) Ozone observation and C2-C5 hydrocarbon observations in the marine boundary layer between $45^{\circ} \mathrm{S}$ and $77^{\circ} \mathrm{S}$. Tellus $B, \mathbf{5 0}, 430-448$.

2 Day, J.A. (1964) Production of droplets and salt nuclei by the bursting of air bubbles films. Q.J. Roy. Meteor. Soc., 90, 72-78.

3 McIntyre, F. (1968) Bubbles. A boundary-layer "microtome" for micron thick sample of liquid surface. J. Chem. Phys., 72, 589-592.

$4 \mathrm{Wu}$, J. (1979) Sea spray in the atmospheric surface layer: review and analysis of laboratory and oceanic results, $J$. Geophys. Res., 84, 1638-1704.

5 Sadiki, M., Quentel, F., Elléouet, C., Huruguen, J.-P., Jestin, J., Andrieux, D., Olier, R. and Privat, M. (2003) Coadsorption at the air/water interface likely explains some pollutants transfer to the atmosphere: benzene and lead case. Atmos. Environ., 37, 3551-3559.

6 Sadiki, M., Quentel, F., Elléouet, C., Olier, R. and Privat, M. (2005) Coadsorption at the air/water interface as source of pollutant transfer to atmosphere. Case study of benzene/cyclohexane traces and lead, Atmos. Environ., 39, 2661-2672.
7 Botelho, C.M.S., Boaventure, R.A.R. and Gonçalves, M.L.S.S. (2002) Interactions of $\mathrm{Pb}$ (II) with particles of a polluted river. Anal. Chim. Acta, 465, 73-85.

8 Adamson, A.W., Physical chemistry of surfaces, WileyInterscience, New-York (1990).

9 Cutting, C.L. and Jones, D.C. (1955) Adsorption of insoluble vapours on water surfaces. J. Chem. Soc., 4067-4075.

10 Shahidzadeh, N., Bonn, D., Ragil, K., Broseta, D. and Meunier, J. (1998) A sequence of two wetting transitions by tuning the Hamaker constant, Phys. Rev. Lett., 80, 3992-3996.

11 Bertrand, E., Hobbs, H., Broseta, D., Indekeu, J., Bonn, D. and Meunier, J. (2000) First-order and critical wetting of alkanes on water. Phys. Rev. Lett., 85, 1282-1285.

12 Bertrand, E., Bonn, D., Broseta, D. and Meunier, J. (1999) Wetting of Hydrocarbon mixtures on water under varying pressure or composition. J. Petrol. Sci. Eng., 24, 221-230.

13 Wan, J. and Tokunaga, T.K. (1998). Measuring partition coefficients of colloids at air-water interfaces. Env. Sci. Technol., 32, 3293-3298.

14 Cahn, J.W. (1977) Critical point wetting. J. Chem. Phys., 66, 3667-3672.

15 Indekeu, J., Ragil, K., Bonn, D., Broseta, D. and Meunier, J. (1999). Wetting of alkanes on water from a Cahn-type theory. J. Stat. Phys., 95, 1009-1043.

16 Rowlinson, J.S. and Widom, B. (1982) Molecular theory of capillarity. Clarendon, Oxford.

17 Yoffe, A. and Heymann, E. (1943). Note on Antonoff's rule. J. Chem. Phys., 47, 409-410.

18 Takii, T. and Mori, Y.H. (1993). Spreading coefficients of aliphatic hydrocarbons on water. J. Colloid Interf. Sci., 161, 31-37.

19 Ross, S. and Becher, P. (1992). J. Colloid Interf. Sci., 149, 575579.

20 Lemlich, R. (1972) Adsorptive bubble methods, in Recent developments in separation science, vol. 1, 113-127, Li, N.N., (Ed.), The Chemical Rubber Co., Cleveland OH.

21 Yaron, B., Calvet, R. and Prost, R. (1996) Soil pollution. Processes and dynamics. Springer, Berlin.

22 Carslow, H.S. and Jaeger, J.C. (1959) Conduction of heat in Solids, 2nd ed. Oxford Clarendon Press, Oxford.

23 Vogt, F., Tacke, M., Jakusch, M. and Mizaikoff, B. (2000) A UV spectroscopic method for monitoring aromatic hydrocarbons dissolved in water. Anal. Chim. Acta, 422,187-198.

24 Norme française NF ISO 11423-1. Qualité de l'eau. Détermination du benzène et de certains dérivés benzéniques par chromatographie en phase gazeuse de l'espace de tête.

25 Lovrič, M. (2002) Stripping voltammetry in Electroanalytical Methods: Guide to experiments and applications, Scholz, F. (Ed.) Springer-Verlag, New York, 191-211.

26 Mc Auliffe, C. (1966) Solubility in water of paraffin, cycloparaffin, olefin, acetylene, cycloolefin, and aromatic hydrocarbons. J. Phys. Chem., 70, 1267-1275.

27 Mc Auliffe, C. (1969) Solubility in water of normal C9 and C10 alkane hydrocarbons. Science, 163, 478-479.

28 Mc Auliffe, C. (1963) Solubility in water of $\mathrm{C}_{1}-\mathrm{C}_{9}$ hydrocarbons. Nature, 200, 1092-1093.

29 Ramos-Gomez, F. and Widom, B. (1980) Noncritical interface near a critical end point II, Physica A, 104, 595-620.

30 Tavan, P. and Widom, B. (1983) Van der Waals model for the surface tension of liquid ${ }^{4} \mathrm{He}$ near the lambda point, Phys. Rev. $B, 27,180-193$. 
31 Widom, B. (1985) Phase equilibrium and interfacial structure Chem. Soc. Rev., 14, 121-140.

32 Nagarajan, N., Webb, W.W. and Widom, B. (1982) Surface tension of two-component liquid mixture near its critical solution point, J. Chem. Phys., 77, 5771-5783.

33 Amara, M., Privat, M., Bennes, R. and Tronel-Peyroz, E. (1991) Experimental study of the critical laws for the surface tensions along the critical isochore, and the coexistence curve and for the liquid-liquid interface. Water-2,5 lutidine system, Europhys. Lett., 16, 153-158.

34 Karad, S., Amara, M., Laouenan, A., Tronel-Peyroz, E., Bennes, R. and Privat, M. (1994) Universal amplitude ratios of surface tensions near a critical point in a liquid binary system: water-2,5 lutidine, J. Chem. Phys., 100, 1498-1502.

35 Massart, D.L., Vandeginste, B.G.M., Buydens, L.M.C., De Jong, S., Lewi, P.J. and Smeyers-Verbeke, J. (1997) Handbook of Chemometrics and Qualimetrics. Part A. Elsevier, Amsterdam, 310.

36 Hamaker, H.C. (1937) The London-Van der Waals attraction between spherical particles. Physica IV-10, 1058-1072.

37 Acharid, A., Quentel, F., Elleouet, C., Olier, R. and Privat, M. (2006) Coadsorption of carbofuran and lead at the air/water interface. Possible occurrence of non-volatile pollutant co-transfer to the atmosphere. Chemosphere, 62, 989-997.

38 Aveyard, R., Saleem, S.M. and Heselden, R. (1977) Desorption of electrolytes at liquid-vapour and liquid-liquid interfaces. J. Chem. Soc. Faraday T., 73, 84-94.

39 Onsager, L. and Samaras, N.N.T. (1934) The surface tension of Debye-Hückel electrolytes. J. Chem. Phys., 2, 528-536.

40 Wagner, C. (1924) Die Oberflächenspannung verdünnter elektrolytlösungen. Physik Z., 25, 474-477.

41 Karraker, K.A. and Radke, C.J. (2002) Disjoining pressure, zeta potential, and surface tension of aqueous non-ionic surfactants/electrolyte solutions: theory and comparison with experiments. Adv. Colloid Interface Sci., 96, 231-264.

42 Jungwirth, P. and Tobias, D.J. (2001) Molecular structure of salt solutions: a new view of the interface with implication for heterogeneous atmospheric chemistry. J. Phys. Chem. B, 105, 10468-10472.

43 ASTDR 1993. Toxicological profile for lead. Final Report of the Agency for Toxic Substances and Disease Registry. Public Health Service, US Department of Health and Human Service (April, 1993).

Final manuscript received in March 2006 